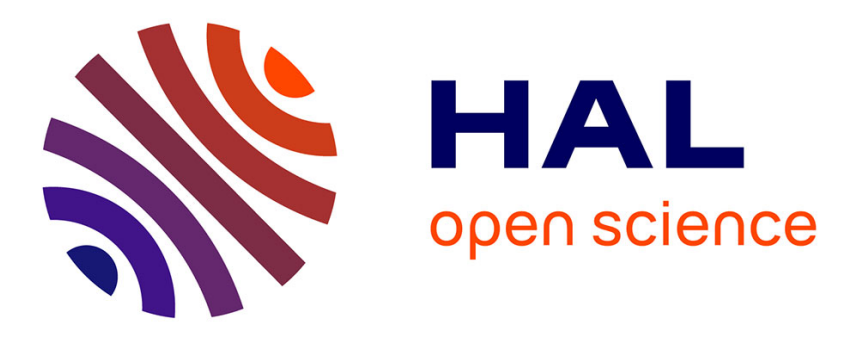

\title{
Human Ccr4-Not complexes contain variable deadenylase subunits
}

Nga-Chi Lau, Annemieke Kolkman, Frederick M.A. van Schaik, Klaas W. Mulder, W.W.M. Pim Pijnappel, Albert J.R. Heck, H.Th. Marc Timmers

\section{- To cite this version:}

Nga-Chi Lau, Annemieke Kolkman, Frederick M.A. van Schaik, Klaas W. Mulder, W.W.M. Pim Pijnappel, et al.. Human Ccr4-Not complexes contain variable deadenylase subunits. Biochemical Journal, 2009, 422 (3), pp.443-453. 10.1042/BJ20090500 . hal-00479183

\section{HAL Id: hal-00479183 \\ https://hal.science/hal-00479183}

Submitted on 30 Apr 2010

HAL is a multi-disciplinary open access archive for the deposit and dissemination of scientific research documents, whether they are published or not. The documents may come from teaching and research institutions in France or abroad, or from public or private research centers.
L'archive ouverte pluridisciplinaire HAL, est destinée au dépôt et à la diffusion de documents scientifiques de niveau recherche, publiés ou non, émanant des établissements d'enseignement et de recherche français ou étrangers, des laboratoires publics ou privés. 


\section{Human Ccr4-Not complexes contain variable deadenylase subunits}

Nga-Chi Lau ${ }^{*}$, Annemieke Kolkman ${ }^{\dagger \neq 1}$, Frederik M.A. van Schaik ${ }^{*}$, Klaas W. Mulder ${ }^{\S}$, W.W. M. Pim Pijnappel ${ }^{* *}$, Albert J.R. Heck ${ }^{\dagger}$ and H.Th. Marc Timmers ${ }^{*}$

*Department of Physiological Chemistry, University Medical Center Utrecht, Universiteitsweg 100, 3584 CG, Utrecht; 'Biomolecular Mass Spectrometry and Proteomics Group, Bijvoet Center for Biomolecular Research, Utrecht University, Sorbonnelaan 16, 3584 CA, Utrecht; "Netherlands Proteomics Centre, Padualaan 8, $3584 \mathrm{CH}$, Utrecht, The Netherlands; ${ }^{\S}$ Epithelial Cell Biology Laboratory, Cancer Research UK Cambridge Research Institute, Li Ka-Shing Centre, Robinson Way, CB2 0RE, Cambridge, United Kingdom

${ }^{1}$ Present address: Department of Metabolic and Endocrine Diseases \& Netherlands Metabolomics Centre, University Medical Centre Utrecht, Lundlaan 6, 3584 EA, Utrecht, The Netherlands.

${ }^{2}$ Address correspondence to: H.Th. Marc Timmers, Department of Physiological Chemistry, University Medical Centre Utrecht, Universiteitsweg 100, 3584 CG, Utrecht, The Netherlands. Tel: +31-88-7568981. Fax: +31-88-7568101.

E-mail: h.t.m.timmers@,umcutrecht.nl

Running title: CNOT7 and CNOT8 form different human Ccr4-Not complexes

Abbreviations used: CNOT, Ccr4-Not; TBP, TATA binding-protein; SCX, strong cation exchange; IPI, International Protein Index; NSAF, normalized spectral abundance factors; GO, Gene Ontology; NUP, nuclear pore proteins. 


\section{SYNOPSIS}

The Ccr4-Not complex is evolutionary conserved and important for regulation of mRNA synthesis and decay. The composition of the yeast complex has been well described. Orthologs of the yeast Ccr4-Not components have been identified in human cells including multiple subunits with mRNA deadenylase activity. In this study, we examine the composition of the human Ccr4-Not complex in an in-depth proteomic approach using stable cell lines expressing tagged CNOT proteins. We find at least four different variants of the human complex, consisting of seven stable core proteins and mutually-exclusive associated mRNA deadenylase subunits. Interestingly, human CNOT4 is in a separate $\sim 200 \mathrm{kDa}$ complex. Furthermore, analyses of associated proteins indicate involvement of Ccr4-Not complexes in splicing, transport and localization of RNA molecules. Taken together, human Ccr4-Not complexes are heterogeneous in composition due to differences in their deadenylase subunits, which may reflect the multi-functionality of these complexes in cellular processes.

Key words: Ccr4-Not, gene expression, transcription regulation, mRNA deadenylation, proteomics. 


\section{INTRODUCTION}

The evolutionary conserved Ccr4-Not complex is important for multiple cellular functions. It is involved in several aspects of the mRNA regulatory pathways (for reviews see $[1,2]$ ). Besides that, the E3 ligase activity of yeast Not4p and human CNOT4 subunits [3, 4] implicates the Ccr4-Not complex in the protein ubiquitination/degradation pathways [3, 5]. Moreover, members of the Ccr4-Not complex are associated with various functions in several species, both in the nucleus and in the cytoplasm. These include involvement in DNA repair and histone methylation in yeast $[6,7]$, in spindle positioning and regulation of microtubule length in C. elegans [8] and in spermatogenesis in mice [9].

The Ccr4-Not complex may influence the synthesis of mRNA molecules in several ways. It has been reported that some yeast Ccr4-Not subunits interact both physically and genetically with TATA-binding protein (TBP), TBP-associated factors (TAFs), and other regulators of TBP binding, indicating direct regulation of gene expression at the core promoter through binding of these basal transcription factors (reviewed in [10]). The Ccr4-Not complex may also influence gene transcription by affecting chromatin structure $[7,11,12]$ or by repressing the nuclear receptor-mediated transcription in a ligand-dependent manner via CNOT1 [13]. Two other human CNOT proteins, CNOT2 and CNOT9/hRcd1/hCAF40, are also implicated in repression of promoter activity [11]. The major repression function of CNOT2 was found in a conserved protein motif called the Not-Box, which is also present at the C-terminus of CNOT3 [11]. Furthermore, the Ccr4-Not complex also plays a direct role in mRNA degradation and aberrant mRNA surveillance (reviewed in [14]). The deadenylase subunits in the complex are responsible for these events. Ccr4p is the major mRNA deadenylase in yeast $[15,16]$, whereas mammalian cells require both CNOT6/hCcr4 and CNOT7/hCAF1 activities for constitutive deadenylation [17].

The composition of the conserved Ccr4-Not complex has been well studied in yeast. Initially, it was identified as physically and functionally separate subcomplexes of Ccr4associated proteins and of Not proteins [18]. Not1p/Cdc39p, Not2p/Cdc36p, Not3p, Not $4 p / M o t 2 p$ and Not5p form the Not module, which regulates transcription initiation and elongation (reviewed in $[1,2]$ ). They were first isolated as repressors of HIS3 gene transcription from a non-canonical TATA element in yeast (reviewed in [10]). The Ccr4 module encompasses the two deadenylases Ccr4p and Caflp in association with Caf40p and Caf130p [19, 20], and is involved in mRNA degradation. Surprisingly, biochemical analyses revealed that the Ccr4 module also binds Not proteins $[19,20]$, resulting in the Ccr4-Not protein complex. Interestingly, the Ccr4p subunit affects transcription both negatively and positively [20]. Orthologs for most of the yeast Ccr4-Not components have been identified in human cells $[21,22]$. Not3p and Not5p are similar yeast proteins, and the human genome contains only one orthologous protein, CNOT3 [21]. In contrast, two human orthologs have been found for yeast Ccr4p (CNOT6/hCcr4a, CNOT6L/hCcr4b) [23] and for yeast Caflp (CNOT7/hCAF1 and CNOT8/hPOP2/CALIF) [21]. These four deadenylases have been identified as components of human Ccr4-Not complexes [23, 24], but the exact composition of the human complex is less well studied.

Here, we determine the composition and the interactors of human Ccr4-Not complexes from stable HeLa cell derivatives, which express epitope-tagged Ccr4-Not subunits at near endogenous levels. We identified the interactors of the purified complexes from these cell lines by mass spectrometry. Strikingly, the CNOT7 and CNOT8 proteins specify the distinct human Ccr4-Not complexes, whereby CNOT6 and CNOT6L associate more tightly with CNOT7 than with CNOT8. Besides the known Ccr4-Not proteins, we invariably and abundantly co-purified TAB182 and C2ORF29 proteins in human Ccr4-Not complexes, indicating that these subunits are novel core complex components. Moreover, our data implicate that association of CNOT4 with the Ccr4-Not complex may be a regulated event. 
Furthermore, additional Gene Ontology analyses of interactors of the complex suggest direct involvement of CNOT7-containing complexes in mRNA splicing, transport and localization, whereas CNOT8-containing complexes seem to play a role in splicing only. Taken together, our findings support the model wherein Ccr4-Not complexes are involved from RNA synthesis in the nucleus to RNA decay in the cytoplasm.

\section{MATERIALS AND METHODS}

\section{Antibodies and immunoblotting}

CNOT1, CNOT2 and CNOT3 antibodies were previously described [13]. Crude sera raised against CNOT8 (described in [21]) were affinity-purified via immunoblotting against a GSTCNOT8 fusion protein and elution from the membrane using $100 \mathrm{mM}$ glycine-HCl pH 2.5. Antibodies directed against CNOT7 (clone 2F6), HA (clone 3F10), alpha-tubulin (DM1A), and TAB182 were obtained from Abnova Corporation, Roche Applied Science, Calbiochem and Novus Biologicals, respectively. The HA (12CA5) antibody was purified from the hybridoma cell line 12CA5 (ATTC).

Protein complexes or extracts were separated on SDS-polyacrylamide gels and transferred onto PVDF membranes. The membranes were blocked using $5 \%(\mathrm{w} / \mathrm{v})$ non-fat milk, probed with primary antibodies overnight at $4{ }^{\circ} \mathrm{C}$ and with HRP-conjugated secondary antibodies for $45 \mathrm{~min}$ at room temperature. Detection was performed by enhanced chemiluminescence according to manufacturer's protocol (ECL, PerkinElmer Life and Analytical Sciences).

\section{cDNA sequences of human CNOT genes}

Human cDNAs of CNOT1 (partial), CNOT2, CNOT3S, CNOT3L, CNOT4N, CNOT4L, CNOT8 and CNOT9 were previously described [11, 21]. cDNA sequences of human CNOT6 ORF was obtained by PCR using pGal4-hCCR4 [25] as a template. Full-length cDNAs of human CNOT7 and CNOT10 were purchased from RZPD, Germany. All cDNA constructs were verified by DNA sequencing.

\section{Generation of stable cell lines}

Human CNOT cDNAs were introduced by GATEWAY cloning [26] into retroviral destination plasmid derived from pBABE-puro carrying N-terminal FlagPreScissionHA-tag (for CNOT2, CNOT3L, CNOT4L, CNOT6, CNOT8, CNOT9, CNOT10) or C-terminal HAPreScissionFlag-tag (for CNOT3S and CNOT7). Details on primer sequences and cloning strategy are available upon request. HeLa S3 cell clones expressing a tagged CNOT protein were obtained by retroviral transduction and puromycin selection. Positive clonal cell lines were identified by immunoblotting using antibodies directed against HA, CNOT2, CNOT3, CNOT7 or CNOT8. HeLa S3 cells with integrated empty pBABE-puro plasmid served as a control.

\section{Overexpression in HEK293T cells}

Human CNOT7 and CNOT8 cDNAs were introduced by GATEWAY cloning into pMT2SM carrying an N-terminal HA-tag. Human embryonic kidney 293T cells were transfected with 3 $\mu \mathrm{g}$ of pMT2SM-HA-CNOT7 or pMT2SM-HA-CNOT8 using FuGene6 according to manufacturer's instructions (Roche Applied Science). Cells were extracted $40 \mathrm{~h}$ posttransfection in lysis buffer (20 mM HEPES-NAOH pH 8.0, 5\% (v/v) glycerol, $150 \mathrm{mM} \mathrm{NaCl}$, $0.5 \mathrm{mM}$ EDTA, $5 \mathrm{mM} \mathrm{MgCl}_{2}, 0.5 \%$ (v/v) NP40, $0.5 \mathrm{mM}$ PMSF, $0.5 \mathrm{mM}$ DTT and complete protease inhibitor cocktail (Roche Applied Science)) and analysed by immunoblotting. 


\section{Cell culture conditions}

HeLa S3 stable cell lines and HEK293T cells were grown in Dulbecco's Modified Eagle's Medium (DMEM, $1 \mathrm{~g} / \mathrm{L}$ glucose) with $10 \%$ (v/v) fetal bovine serum, L-glutamine, penicillin/streptomycin (and $1 \mu \mathrm{g} / \mathrm{mL}$ puromycin for HeLa S3). HeLa S3 cells were grown in bioreactors in Modified Eagle Medium with Hank's balanced salt solution (MEM), 10\% (v/v) fetal bovine serum, L-glutamine, non essential amino acids, penicillin/streptomycin and 1 $\mu \mathrm{g} / \mathrm{mL}$ puromycin. All reagents were obtained from Cambrex, except for puromycin (Sigma).

\section{Protein extraction and affinity purification}

HeLa cells were lysed by douncing in hypotonic lysis buffer $(10 \mathrm{mM}$ HEPES-NAOH pH 8.0, $10 \mathrm{mM} \mathrm{KCl}, 1.5 \mathrm{mM} \mathrm{MgCl} 2,0.5 \mathrm{mM}$ DTT and $0.5 \mathrm{mM}$ PMSF). Proteins were extracted in $1 / 3$ volume of $4 \mathrm{x}$ extraction buffer ( $80 \mathrm{mM}$ HEPES-NAOH $\mathrm{pH} 8.0,50 \%$ (v/v) glycerol, 1.68 $\mathrm{M} \mathrm{NaCl}, 0.8 \mathrm{mM}$ EDTA, $3 \mathrm{mM} \mathrm{MgCl} 2,1 \mathrm{mM}$ DTT and 4\% (v/v) protease inhibitor cocktail (Sigma)), bringing the ionic strength to $420 \mathrm{mM} \mathrm{NaCl}$. Lysates were centrifuged at $168,000 \mathrm{~g}$ for $45 \mathrm{~min}$ at $4^{\circ} \mathrm{C}$. Supernatants were dialysed overnight against dialysis buffer $(20 \mathrm{mM}$ HEPES-NAOH pH 8.0, 20\% (v/v) glycerol, $100 \mathrm{mM} \mathrm{KCl,} 0.2$ mM EDTA, $0.5 \mathrm{mM}$ PMSF and $0.5 \mathrm{mM}$ DTT).

Dialysed extracts $(10-20 \mathrm{mg} / \mathrm{mL})$ were incubated for 5 hours with $1 / 20$ volume of M2 affinity resin (Sigma) pre-equilibrated in BC150 buffer (40 mM Tris- $\mathrm{HCl} \mathrm{pH} 7.9,20 \%$ (v/v) glycerol, $150 \mathrm{mM} \mathrm{KCl}, 0.2 \mathrm{mM}$ EDTA and $0.1 \%$ (v/v) NP40). Resin was subsequently washed with 60 column volumes of BC300 buffer $(20 \mathrm{mM}$ Tris- $\mathrm{HCl} \mathrm{pH} \mathrm{7.9,20 \% (v/v)}$ glycerol, $300 \mathrm{mM} \mathrm{KCl}, 0.2 \mathrm{mM}$ EDTA and $0.1 \%$ (v/v) NP40). Proteins were eluted twice with two column volumes of BC300 buffer containing $200 \mu \mathrm{g} / \mathrm{mL}$ FLAG peptide (Sigma). Flag elution fractions were pooled and subsequently incubated overnight with HA affinity resin pre-equilibrated in $\mathrm{BC} 300$ buffer. HA affinity resin was prepared by covalently binding of HA (12CA5) antibody to Dynabeads protein A (Dynal Biotech) using dimethyl pimelimidate dihydrochloride (Pierce) according to manufacturer's instructions. After overnight incubation resin was washed with 15 resin volumes of BC300 buffer. Bait and interacting proteins were eluted for $20 \mathrm{~min}$ at $30^{\circ} \mathrm{C}$ with $2 \mathrm{mg} / \mathrm{mL} \mathrm{HA}$ peptide (SigmaGenosys), followed by precipitation [27] and identification by mass spectrometry or separated on $4-12 \%$ Bis-Tris gel (NuPAGE, Invitrogen) for silver stain detection.

For affinity purification at lower salt conditions, cells were lysed in hypotonic lysis buffer, dounced and proteins were extracted in $1 / 3$ volume of $4 x$ extraction buffer containing 600 $\mathrm{mM} \mathrm{NaCl}$, bringing the ionic strength to $150 \mathrm{mM} \mathrm{NaCl}$, followed by overnight dialysis. Extracts were purified via the Flag-tag as described above using buffers containing $150 \mathrm{mM}$ $\mathrm{NaCl}$. Purified complexes were subjected to immunoblotting.

\section{Mass spectrometry}

Precipitated Ccr4-Not complexes were dissolved in $8 \mathrm{M}$ urea in $50 \mathrm{mM}$ ammoniumbicarbonate $\mathrm{pH} 8.0$, and incubated with $1 \mu \mathrm{g}$ of LysC (Roche Diagnostics) for $4 \mathrm{~h}$ at $37^{\circ} \mathrm{C}$. Following reduction and alkylation using $2 \mathrm{mM}$ DTT and $4 \mathrm{mM}$ iodoacetamide, respectively, the sample was diluted to $2 \mathrm{M}$ urea with $50 \mathrm{mM}$ ammonium bicarbonate $\mathrm{pH} 8.0$, and incubated overnight with $2 \mu \mathrm{g}$ of trypsin at $37^{\circ} \mathrm{C}$. Protein digests were desalted using a small plug of $\mathrm{C} 18$ material (3M Empore $\mathrm{C}_{18}$ extraction disk) packed into a GELoader Tip similar to as previously described [28]. The eluate was dried completely by vacuum centrifugation and subsequently reconstituted in $20 \%(\mathrm{v} / \mathrm{v})$ acetonitrile, $0.05 \%(\mathrm{v} / \mathrm{v})$ formic acid.

Strong cation exchange (SCX) was performed using two Zorbax BioSCX-Series II columns (i.d., $0.8 \mathrm{~mm}$; 1, $50 \mathrm{~mm}$; particle size, $3.5 \mu \mathrm{m}$ ), a Famos autosampler (LCpackings, Amsterdam, The Netherlands), a Shimadzu LC-9A binary pump, and a SPD-6A UV-detector 
(Shimadzu, Tokyo, Japan). After injection of the peptides, the first 10 min were run isocratically at $100 \%$ solvent A $(0.05 \%(\mathrm{v} / \mathrm{v})$ formic acid in $8 / 2(\mathrm{v} / \mathrm{v})$ water/acetonitrile $\mathrm{pH}$ $3.0)$, followed by a linear gradient of $1.3 \% \mathrm{~min}^{-1}$ solvent $\mathrm{B}(500 \mathrm{mM} \mathrm{NaCl}$ in $0.05 \%(\mathrm{v} / \mathrm{v})$ formic acid in $8 / 2(\mathrm{v} / \mathrm{v})$ water/acetonitrile $\mathrm{pH} 3.0)$. A total number of $24 \mathrm{SCX}$ fractions (1 min each, i.e., $50 \mu \mathrm{L}$ elution volume) were manually collected and dried in a vacuum centrifuge. These residues were reconstituted in $20 \mu \mathrm{L}$ of $0.1 \mathrm{M}$ acetic acid and approximately half of this solution was analysed by on-line nanoflow liquid chromatography FT-ICR-MS/MS as previously described [29].

Protein identification was processed as described [29] using International Protein Index (IPI) human (release 3.24, 66921 sequences entries) and Scaffold software package version 2.1 (www.proteomesoftware.com) with confidence level of $95 \%$ for peptide identification and 99\% for protein identification. Unique peptide counts of the identified Cer4-Not components are given in Supplemental Table 1. Total number of spectral counts for each component was divided by its mass (in Daltons) and converted into normalized spectral abundance factors (NSAF) as described in [30].

The data associated with this manuscript may be downloaded from ProteomeCommons.org Tranche, https://proteomecommons.org/tranche/, using the following hash: EKYhwbHIfbis7ocZqc+18hDwmT7WZvSLAWp1dVKjhBWF9W2Ubb8IEScvJqhwZMJQM YBpaPATHB/yVedmNIPMeNej10cAAAAAAAADPw==.

\section{Bioinformatics}

IPI numbers of identified proteins (by at least two unique peptides, no peptides in a control purification, excluding keratins) were converted to gene symbols using PICR (http://www.ebi.ac.uk/Tools/picr/), DAVID (http://david.abcc.ncifcrf.gov/home.jsp) and UniProt (http://www.uniprot.org/). Some IPI numbers could not be mapped to a gene and were renamed $\mathrm{nm} \_\mathrm{x}$ (non-mapped_x). A list of genes for each purification was submitted to GeneTrail ([31]; http://genetrail.bioinf.uni-sb.de/index.php) using all human genes as a reference set. Gene Ontology (GO) term overrepresentation was assessed using a hypergeometric test with false discovery rate (FDR) adjustment and a significance threshold of 0.05 . In addition, a minimum number of two genes per GO-term were required for a GOterm to be included in the analyses. All statistically significant overrepresented GO-terms were collected and a matrix was generated consisting of the GO-terms and their p-values for each of the purifications. P-values were transformed to a $\log _{2}$ scale to reduce effects of abnormal data distribution during subsequent analyses. Missing values were converted to zero. Matrices containing $\log _{2}$ transformed $p$-values of overrepresented GO-terms were loaded into the clustering module (GenesisWeb) of CARMAweb ([32]; https://carmaweb.genome.tugraz.at/carma/) to generate hierarchically clustered heatmaps according to Pearson correlation. The HomoMINT database (download date 10 June 2009) is used to obtain interologs (http://mint.bio.uniroma2.it/HomoMINT/Welcome.do).

\section{Yeast two-hybrid}

The pSH18-34 plasmid, pJG4-5 and pEG202-NLS vectors, B42-CNOT1C, B42-CNOT2, B42-CNOT4N and B42-CNOT8 (B42-CALIF) were previously described $[12,21]$. Other yeast plasmids were created using full-length cDNA sequences of human CNOT proteins, except for B42-CNOT7. This latter construct was obtained by insertion of a murine CNOT7 fragment into pJG4-5, derived from pGal4-mCAF1 [33] containing an extended N-terminal part of eleven amino acids and a point mutation to resemble human protein sequences. Details on the cloning strategy are available upon request. Yeast strain EGY48 (MATa his3 trp1 ura3 LEU2::pLexAop (x6)-LEU2) was used for this assay [21]. Interactions were visualised on $\mathrm{X}$ gal containing plates at $30^{\circ} \mathrm{C}$. 


\section{Gel filtration}

Cell extracts of the CNOT4L-tagged HeLa S3 stable cell line was prepared as described above with the following adaptations: hypotonic lysis buffer also contained $2 \%(\mathrm{v} / \mathrm{v})$ protease inhibitor cocktail (Sigma), cells were lysed by syringe douncing and proteins were extracted by addition of 1 volume of extraction buffer $(20 \mathrm{mM}$ HEPES-NAOH pH 8.0, 20\% (v/v) glycerol, $300 \mathrm{mM} \mathrm{NaCl}, 0.4 \mathrm{mM}$ EDTA, $1.5 \mathrm{mM} \mathrm{MgCl}_{2}, 0.2 \%$ (v/v) NP40 and $0.5 \mathrm{mM}$ DTT). Extracts were analysed on a Superose 6 HR 10/30 column (GE Healthcare) in $20 \mathrm{mM}$ HEPES-NAOH pH 7.9 at $4^{\circ} \mathrm{C}, 150 \mathrm{mM} \mathrm{NaCl}, 10 \mu \mathrm{M} \mathrm{ZnCl}_{2}, 1 \mathrm{mM}$ DTT, $0.5 \mathrm{mM}$ PMSF, Leupeptin, Aprotinin and Pepstatin A buffer, followed by immunoblot analyses of the 0.4 mL-fractions.

\section{RESULTS}

Stable HeLa S3 cell lines with Flag/HA-tagged CNOT proteins at endogenous conditions The human Ccr4-Not complex is biochemically less well-defined than its yeast counterpart $[18,23,24]$. To obtain more insight into the composition of the human complex, we generated HeLa cell lines that stably express Flag/HA-tagged versions of human Ccr4-Not (CNOT) subunits. The expression levels of these tagged proteins within the distinct cell lines were similar with exception of the higher expressed CNOT9 (Figure 1A). Immunoblot analyses using specific antibodies against CNOT2, CNOT7 or CNOT8 showed near endogenous expression levels of the tagged protein (Figure 1B). Immunoprecipitation of tagged CNOT3 or CNOT9 resulted in co-purification of the known subunits of Ccr4-Not complex like CNOT2, CNOT3 and CNOT7 (Figure 1C), indicating that the tagged proteins are integrated into larger protein complexes. Notably, similar amounts of CNOT2 and CNOT7 could be coimmunoprecipitated from cell extracts containing tagged CNOT3L or the higher expressed tagged CNOT9 (Figure 1A and 1C). This indicates that the higher expression levels of CNOT9 did not disrupt normal Ccr4-Not complex formation. In addition, silver stain analysis of large-scale affinity purifications of CNOT3L, CNOT7 and CNOT9 cell lines revealed copurification of other proteins, which were identified as Ccr4-Not complex subunits by mass spectrometry (Figure 1D and Table 1). Thus, the generated HeLa S3 cell lines express their epitope-tagged proteins at near endogenous levels and are integrated into endogenous Ccr4Not complexes.

\section{Human Ccr4-Not complexes differ in deadenylase subunits}

To analyse the composition of human Ccr4-Not complexes from different entry points, tagged HeLa S3 cells were grown in bioreactors for large-scale affinity purifications of Ccr4-Not complexes, which were subjected to in-solution tryptic digestion, strong cation exchange (SCX) fractionation and nano-reversed phase liquid chromatography coupled to high resolution mass spectrometry (LTQ-FT-ICR) analyses. Table 1 summarizes the results in normalized spectral abundance factors (NSAFs) to indicate relative abundances of co-purified proteins among the individual CNOT purifications [30] (Supplemental Figure 1 is a coloured representative of Table 1). In this study, we define a core subunit of the Ccr4-Not complex as being identified by mass spectrometry with at least a NSAF value of 0.4 in all Ccr4-Not purifications and with no peptides in a control purification. We define a subunit as variable when the NSAF value is at least 0.4 but not in all purifications. According to these criteria, the deadenylases CNOT6, CNOT6L, CNOT7 and CNOT8 are variable subunits within the human complex and CNOT4 E3 ligase is not an integral subunit of human Ccr4-Not (Table 1). CNOT1, CNOT2, CNOT3, CNOT9, CNOT10 and the new interactors TAB182 and 
C2ORF29 are identified as core subunits of the human complexes (Table 1). The C2ORF29 protein is predicted from the human genome sequence with no known function and TAB182 has been described as a binding protein for tankyrase 1, which plays a role in telomere length and mitotic spindle composition [34, 35]. Immunoblot analysis showed that a small but significant proportion of cellular TAB182 co-purified with CNOT3L and CNOT9 complexes (Figure 1E), confirming that TAB182 is a stable interactor of the human Ccr4-Not complex. Unfortunately, no antibodies against C2ORF29 are available precluding confirmation by Western blotting.

The detailed mass spectrometry results revealed several interesting properties of human Ccr4-Not complexes (Table 1). In particular, the variable deadenylase subunits show interesting differences among the tagged CNOT6, CNOT7 and CNOT8 purifications (given in bold in Table 1). No significant CNOT7 was detected in CNOT8 purifications and vice versa, suggesting that CNOT7 and CNOT8 are present in distinct Ccr4-Not complexes. CNOT6 and CNOT6L were identified in CNOT7 purifications, whereas they were absent from CNOT8 purifications, suggesting that CNOT6/6L proteins stably interact with CNOT7 but not with CNOT8. No CNOT6L could be identified in two CNOT6 purifications (Table 1 and Tranche database), indicating mutually exclusiveness of these subunits as expected from their distinct functions; CNOT6L, but not CNOT6, impairs cell growth [23]. It is interesting to note that the deadenylases CNOT6, CNOT6L, CNOT7 and CNOT8 are relatively lower represented in the purifications than the other CNOT proteins, except when they are the bait of the purification. Moreover, the deadenylase subunits could not be detected in CNOT10-purified complexes. This could, however, be due to the lower amounts of recovered proteins in this purification. Importantly, Ccr4-Not complexes obtained via other tagged subunits inversely identified CNOT10. Furthermore, comparison of full-length CNOT3L (residues 1-753) to CNOT3S (residues 1-609) lacking the Not-Box showed that the smaller isoform did not interact with any Ccr4-Not subunit. This indicates that the Not-Box is required for stable interaction of CNOT3 with the rest of the complex $[11,21,36]$. Finally, human CNOT4 could hardly be detected in purified Ccr4-Not complexes in contrast to yeast $[18,20]$, indicating that CNOT4 is not stably integrated within human complexes. In conclusion, purified Ccr4-Not complexes from human cells using seven different entry points indicate the existence of several Ccr4-Not complexes differing only in the deadenylase subunits CNOT6, CNOT6L, CNOT7 and CNOT8.

\section{Analyses of substoichiometric interactors suggest Ccr4-Not functioning in RNA splicing and nuclear export}

Besides subunits of Ccr4-Not complexes, the mass spectrometry data identified 498 proteins with at least two unique peptides and no peptides in a control purification. These proteins have low NSAF values and are not present in all purifications. We propose that these 498 proteins represent substoichiometric interactors of tagged Ccr4-Not proteins (Supplemental Table 2). About $47 \%$ of these associated proteins were shared among the deadenylase subunits and the other CNOT proteins, indicating that almost half of them bind both the deadenylases as well as transcriptional Ccr4-Not subunits. In addition, 70\% of the interactors were co-purified by at least two CNOT proteins, $44 \%$ by at least three Ccr4-Not components, and $26 \%$ by at least four subunits. Importantly, these proteins were not identified in a control purification, suggesting that these substoichiometric interactors are relevant for the function of the Ccr4-Not complex. We searched the HomoMINT database [37] for interologs to find seven interactors shared between our MS/MS-based human dataset and the yeast dataset. These shared interologs are rather diverse in function and we find only a few peptides of each interactor (Supplemental Table 3). 
To gain insight into the biological functions of human Ccr4-Not complexes, Gene Ontology (GO) term overrepresentation analyses were performed with the CNOT subunits and the substoichiometric Ccr4-Not interactors. The results are given in Supplemental Figure 2 of which the interesting clusters are represented in Figure 2. Clusters containing gene transcription and RNA stability related terms were identified (Figure 2A and 2B), indicating that expected functions of Ccr4-Not can be retrieved from this data analyses. Interestingly, many subunits give association with mRNA splicing (Figure 2C), mRNA transport and localization (Figure 2D), except for CNOT6 and CNOT8, respectively. Moreover, CNOT6, CNOT8 and CNOT10 have low probability to interact with the nuclear envelope (Figure 2E and $2 \mathrm{~F}$ ), suggesting that these proteins may not be involved directly in nuclear mRNA export. Most Ccr4-Not subunits co-purified proteins of the nuclear pore complex (NUPs) located at the nuclear basket, the cytosolic filaments and inside the nuclear membrane (Supplemental Figure 3). The majority of the NUPs inside the nuclear membrane were co-purified. However, more peptides were retrieved from the identification of NUPs on the cytosolic or nuclear membrane side (Supplemental Figure 3). This suggests that the Ccr4-Not complex may interact with the intact nuclear pore complex. Thus, GO-term overrepresentation of substoichiometric interactors indicates physical interactions of human Ccr4-Not complexes with proteins involved in mRNA processing and nuclear export, suggesting involvement of Ccr4-Not in those cellular processes.

\section{CNOT1 binds CNOT4, and CNOT6 binds CNOT8 in yeast two-hybrid assays}

To further explore the composition of human Ccr4-Not complexes, we investigated binary interactions between known components of the complex by yeast two-hybrid interaction experiments [38]. Instead of using full-length CNOT1 protein, this large protein (2376 residues) was split up into smaller fragments (Figure 3A). Positive binary interactions between a B42-fusion and a LexA-fusion CNOT protein in yeast are shown in Figure 3B. The majority of the subunits - except for CNOT3L and CNOT6 - interacted with the C-terminal part of CNOT1 (residues 648-2376). This is in concordance with earlier findings that a shorter C-terminal CNOT1 (CNOT1C: 1106-2376) interacts with CNOT2, CNOT4N and CNOT8 [21]. In addition, CNOT2 and CNOT9 interacted with the N-terminus of CNOT1 (residues 1716). These observations indicate a scaffold function for CNOT1 as suggested previously for its yeast ortholog Not1p [18]. Moreover, none of the examined subunits interacted with themselves, suggesting that they occur as monomers. Interestingly, CNOT3L only interacted with CNOT2 (Figure 3B), suggesting that CNOT3 integrates within the Ccr4-Not complex by CNOT2 interaction. Furthermore, CNOT6 interacted with CNOT7 and vice versa. Also, CNOT6 showed a binary interaction with CNOT8 in this assay, whereby the reciprocal interaction could not be performed due to self-activation properties of LexA-CNOT8. It is interesting to note that CNOT4N and CNOT1L also interacted in this assay.

\section{CNOT4 resides in a smaller complex outside the human Ccr4-Not complex}

To further investigate the association of CNOT4 with the Ccr4-Not complex, we have generated a cell line that stably expresses the largest isoform of CNOT4, CNOT4L [21]. Extracts of these cells were separated by gel filtration under physiological salt conditions and subjected to immunoblot analyses (Figure 4). Tagged CNOT4L protein resided in a $\sim 200 \mathrm{kDa}$ complex, while the subunits CNOT1, CNOT3, CNOT7 and CNOT8 co-eluted in fractions corresponding to 1.2 MDa. In addition, a small fraction of CNOT8 and of CNOT2 was seen at a single protein size (data not shown). It would be interesting to determine the composition of the $\sim 200 \mathrm{kDa}$ complex. However, the low abundance of this complex precluded our first attempts in this direction (data not shown) 
Taken together, gel filtration analyses confirm the mass spectrometry results that CNOT4 is not stably interacting with human Ccr4-Not components, but rather forms a distinct protein complex in human cells. The identity of CNOT4-associated subunits could reveal how interaction of CNOT4 with the Ccr4-Not complex would be regulated.

\section{CNOT6 interacts stronger with CNOT7 than with CNOT8}

To further analyse the interactions among the four human deadenylases, we first determined the specificity of antibodies directed against CNOT7 or against CNOT8 as these deadenylases are for $75 \%$ identical in their primary structure. Upon transient overexpression of CNOT7 and/or CNOT8, the CNOT7 antibodies specifically recognized CNOT7 (Figure 5A, upper left panel), while antibodies directed at CNOT8 (middle left panel) detected both CNOT7 (upper band) and CNOT8 (lower band). The CNOT7 antibodies also specifically recognized endogenous CNOT7 (Figure 5A, right upper panel), whereas the CNOT8 antibodies detected endogenous CNOT8 but also displayed considerable background bands (Figure 5A, right middle panel). Thus, the specificity of CNOT7 antibodies is exclusive for CNOT7 proteins, and antibodies directed against CNOT8 detect both CNOT7 and CNOT8 subunits, which can be distinguished by different mobility in protein gels.

Immunoblot analyses using these antibodies showed the absence of CNOT7 proteins in CNOT8-purified complexes, and no CNOT8 proteins could be detected in CNOT6- and CNOT7-purified complexes (Figure 5B). As expected, both CNOT7 and CNOT8 were present in CNOT2-purified complexes (Figure 5B). These observations confirm the mass spectrometry results (Table 1 and Supplemental Table 1) of the mutually exclusiveness of CNOT7 and CNOT8 within Ccr4-Not complexes. In addition, the interaction between CNOT6 and CNOT8 was further studied by immunoblot analyses using the described antibodies (Figure 5C). Yeast two-hybrid showed CNOT6 binding to CNOT8, whereas mass spectrometry results did not reveal CNOT6 in CNOT8 complexes and vice versa (Table 1 and Supplemental Table 1). One possibility is the loss of CNOT6-CNOT8 interaction during the two-step purification prior to mass spectrometry identification. To address this hypothesis, one-step Flag purifications of Ccr4-Not complexes were performed under low or high salt concentrations (Figure 5C). The association of CNOT7 within CNOT6- or CNOT2-purified complexes was not sensitive to salt, indicating strong binding of CNOT7 to these complexes. Notably, CNOT8 could be detected just above background in CNOT6-purified complexes under low salt conditions only, whereas CNOT8 was clearly detectable in CNOT2-purified complexes independent of the salt concentration. Together, this indicates that the interaction between CNOT6 and CNOT8 is sensitive to the salt concentration. Unfortunately, the lack of CNOT6 antibodies does not allow us to perform the converse experiment using CNOT7- and CNOT8-purified complexes. In conclusion, these experiments confirm that CNOT7 and CNOT8 deadenylases are mutually-exclusive subunits of human Ccr4-Not complexes, whereby CNOT6 seems to interact less stable with CNOT8 than with CNOT7.

\section{DISCUSSION}

In this study, we investigated the composition and the protein interactors of human Ccr4-Not complexes by an in-depth proteomic effort. We have defined the core proteins of human Ccr4-Not complexes including two new subunits, TAB182 and C2ORF29. These complexes are heterogeneous in composition by their associated mRNA deadenylase subunits, which may reflect functional differences in coupling mRNA synthesis and processing to nuclear export. Size fractionation experiments showed that the human CNOT4 E3 ligase resides in a separate $\sim 200 \mathrm{kDa}$ protein complex. Interestingly, yeast two-hybrid assay displayed a 
CNOT4-interaction with the scaffold protein CNOT1. Moreover, CNOT3 requires its NotBox to integrate into the human Ccr4-Not complex via CNOT2. Taken together, our findings define the composition of human Ccr4-Not complexes and have revealed the co-existence of multiple Ccr4-Not complexes differing in their deadenylase subunits.

\section{Defining the human Ccr4-Not complexes}

In the past, the components and the composition of the yeast Ccr4-Not complex has been revealed by a variety of biochemical purifications and genetic experiments [18-20]. Gel filtration of the yeast complex revealed two peaks of 1.9 MDa and 1.0 MDa [18, 19]. In contrast, we observed a rather broad peak for the human complex around 1.2 MDa (Figure 4). Gavin and colleagues have purified the human Ccr4-Not complex via a TAP-tagged version of CNOT2, but they failed to identify CNOT3, CNOT4 and CNOT6L as Ccr4-Not components [39]. Our mass spectrometry results indicate a core of seven subunits for human Ccr4-Not complexes consisting of CNOT1, CNOT2, CNOT3, CNOT9, CNOT10, TAB182 and C2ORF29 with the associated deadenylases CNOT6, CNOT6L, CNOT7 and CNOT8 (Figure 6). The combined molecular weight of these components into the human complex is $\sim 0.9 \mathrm{MDa}$, which corresponds with the gel filtration results (Figure 4).

The overall composition of human Ccr4-Not complexes is similar to the yeast complex [19]. We found that several CNOT proteins (CNOT2, CNOT7, CNOT8 and CNOT9) interact with the largest subunit of the complex, CNOT1 (Figure 3B), indicating that this protein acts as a scaffold similar to yeast Not1p. However, we noted several differences between the human and yeast Ccr4-Not complexes. First, human cells contain multiple deadenylases, which have different binding properties. Our salt titration experiments indicate that CNOT6 interacted less stably with CNOT8 compared to CNOT7 (Figure 5C). Notably, CNOT6 only showed binary interactions with CNOT7 and CNOT8 in a yeast two-hybrid assay (Figure 3B), indicating that these two deadenylases link CNOT6 to the rest of the Ccr4-Not complex as proposed [21, 40]. Secondly, CNOT3 did not bind directly to CNOT1 as reported for the yeast Ccr4-Not complex [18, 19], but it did bind CNOT2 (Figure 3B). Moreover, mass spectrometry analyses showed that CNOT3 lacking the Not-Box (CNOT3S) could not interact with any Ccr4-Not subunits (Table 1). This suggests that CNOT3 integrates in the human complex via Not-Box mediated interaction with CNOT2. Finally, in contrast to yeast Not4p $[18,19]$, CNOT4 was not stably integrated in human Ccr4-Not complexes (Table 1 and Figure 4), but it could interact with the scaffold protein CNOT1 in a yeast two-hybrid assay (Figure 3B). In addition, human CNOT4 is a true ortholog of yeast NOT4 as it can complement not4A not5 $\triangle$ lethality in yeast [21]. This suggests that the CNOT4 interaction with the Ccr4-Not complex may be a regulated event in human cells. Possibly, association of a core subunit precludes interaction of CNOT4 with CNOT1. It is also possible that interactors of CNOT4 or post-translational modifications are involved in restricting CNOT4 integration into the human complex. A model of the distinct Ccr4-Not complexes in human cells is depicted in Figure 6.

\section{Multiple human Ccr4-Not complexes differing in mRNA deadenylase subunits}

Yeast Ccr4-Not complex harbours two deadenylases Ccr4p and Caflp, of which Caflp is dispensable for Ccr4p deadenylase activity $[15,16]$. Human cells contain two orthologs of yeast Ccr $4 \mathrm{p}, \mathrm{CNOT} / \mathrm{hCcr} 4 \mathrm{a}$ and the recently identified CNOT6L/hCcr4b, and two homologous Caflp orthologs, CNOT7 and CNOT8. All these Ccr4p/Caflp orthologs display in vitro 3' -5 ' poly(A) exoribonuclease activity [23, 41]. Our results revealed that the highly homologous CNOT7 and CNOT8 proteins are in separate complexes (Table 1 and Figure 5B). Only a small portion of CNOT8-containing complexes harbour CNOT6, which seems to be 
less stable than the CNOT6-interaction in CNOT7-containing complexes (Figure 5C). It is plausible that CNOT7 and CNOT8 are competing for the same binding site on CNOT1 allowing association of either CNOT7 or CNOT8. In addition, CNOT6 and CNOT6L may also be mutually-exclusive as no unique peptides of CNOT6L were found in two purifications of CNOT6-tagged complexes (Table 1 and Tranche database). This is also supported by earlier data showing that CNOT6L is functionally distinct from CNOT6 [23]. Furthermore, our data showed co-purification of CNOT6-CNOT7 and CNOT6L-CNOT7 (Table 1), which was previously reported in a transient overexpression approach [40]. Thus, all these findings support a model of distinct Ccr4-Not complexes allowing only a single Ccr $4 p$ and a single Caflp ortholog (Figure 6). It is interesting to note that the substrate specificity for the enzymatic activity of CNOT7 and CNOT8 is different [41], which may allow differential regulation of mRNA degradation.

\section{Human Ccr4-Not complexes play various roles in mRNA synthesis to degradation}

GO-term overrepresentation analyses of associated Ccr4-Not proteins provided insight in the functional roles of human Ccr4-Not complexes such as in gene transcription and RNA stability (Figure 2A and 2B). A role in mRNA transport and localization has also been indicated for CNOT7- but not for CNOT8-containing complexes (Figure 2D-F). Indeed, multiple NUPs interacted with all tested CNOT proteins, except for CNOT8 (Supplemental Figure 3). Interestingly, CNOT6 and CNOT7 proteins were reported to shuttle between the nucleus and cytoplasm [40]. In general, more peptides were retrieved from nuclear and cytosolic NUPs than for NUPs within the nuclear envelope (Supplemental Figure 3). This may indicate a shuttling function of the Ccr4-Not complex, which may link its nuclear and cytosolic functions. In addition, involvement in mRNA splicing is also suggested by GO-term overrepresentation analyses, which included the CNOT8-containing complexes (Figure 2C). Taken together, these findings support the view that human Ccr4-Not complexes can influence the mRNA metabolism at multiple levels.

In conclusion, our study provides comprehensive composition analyses of Ccr4-Not complexes in human cells. We identified multiple Ccr4-Not complexes differing in their deadenylase subunits and in complex stability. Our proteomic analyses provide a platform for further functional studies of human Ccr4-Not complexes defining the regulation of mRNA metabolism by this evolutionary conserved protein complex.

\section{ACKNOWLEDGEMENTS}

We thank Dr. M.A. Collart for the gift of CNOT1 antibody, Dr. V.J. Bardwell for the partial CNOT1 cDNA constructs, and Dr. T. Aoki for the gift of CNOT3 antibody. We also thank Fred Koch for technical assistance in generating and purifying HeLa S3 stable cell line expressing CNOT8, and Danny Navarro for the submission of the Scaffold data to the ProteomeCommons/Tranche database. We are grateful to Dr. P. Kemmeren for bioinformatic assistance. We thank members of the Timmers and Heck laboratories for discussions, and Dr. Nikolay Outchkourov and Loes van de Pasch for critical reading of this manuscript. This work was supported by the Netherlands Proteomics Centre. K.W.M. received a grant of the European Commission 7th Framework Program Marie Curie Fellowship [PIEF-GA-2008220642] 


\section{REFERENCES}

1 Collart, M. A. and Timmers, H. T. (2004) The eukaryotic Ccr4-not complex: a regulatory platform integrating mRNA metabolism with cellular signaling pathways? Prog Nucleic Acid Res Mol Biol. 77, 289-322

2 Denis, C. L. and Chen, J. (2003) The CCR4-NOT complex plays diverse roles in mRNA metabolism. Prog Nucleic Acid Res Mol Biol. 73, 221-250

3 Albert, T. K., Hanzawa, H., Legtenberg, Y. I., de Ruwe, M. J., van den Heuvel, F. A., Collart, M. A., Boelens, R. and Timmers, H. T. (2002) Identification of a ubiquitin-protein ligase subunit within the CCR4-NOT transcription repressor complex. Embo J. 21, 355-364

4 Mulder, K. W., Inagaki, A., Cameroni, E., Mousson, F., Winkler, G. S., De Virgilio, C., Collart, M. A. and Timmers, H. T. (2007) Modulation of Ubc4p/Ubc5p-mediated stress responses by the RING-finger-dependent ubiquitin-protein ligase Not4 $p$ in Saccharomyces cerevisiae. Genetics. 176, 181-192

5 Panasenko, O., Landrieux, E., Feuermann, M., Finka, A., Paquet, N. and Collart, M. A. (2006) The yeast Ccr4-Not complex controls ubiquitination of the nascent-associated polypeptide (NAC-EGD) complex. J Biol Chem. 281, 31389-31398

6 Mulder, K. W., Winkler, G. S. and Timmers, H. T. (2005) DNA damage and replication stress induced transcription of RNR genes is dependent on the Ccr4-Not complex. Nucleic Acids Res. 33, 6384-6392

7 Mulder, K. W., Brenkman, A. B., Inagaki, A., van den Broek, N. J. and Timmers, H. T. (2007) Regulation of histone H3K4 tri-methylation and PAF complex recruitment by the Ccr4-Not complex. Nucleic Acids Res. 35, 2428-2439

8 DeBella, L. R., Hayashi, A. and Rose, L. S. (2006) LET-711, the Caenorhabditis elegans NOT1 ortholog, is required for spindle positioning and regulation of microtubule length in embryos. Mol Biol Cell. 17, 4911-4924

9 Nakamura, T., Yao, R., Ogawa, T., Suzuki, T., Ito, C., Tsunekawa, N., Inoue, K., Ajima, R., Miyasaka, T., Yoshida, Y., Ogura, A., Toshimori, K., Noce, T., Yamamoto, T. and Noda, T. (2004) Oligo-astheno-teratozoospermia in mice lacking Cnot7, a regulator of retinoid X receptor beta. Nat Genet. 36, 528-533

10 Collart, M. A. (2003) Global control of gene expression in yeast by the Ccr4-Not complex. Gene. 313, 1-16

11 Zwartjes, C. G., Jayne, S., van den Berg, D. L. and Timmers, H. T. (2004) Repression of promoter activity by CNOT2, a subunit of the transcription regulatory Ccr4-not complex. $\mathrm{J}$ Biol Chem. 279, 10848-10854

12 Jayne, S., Zwartjes, C. G., van Schaik, F. M. and Timmers, H. T. (2006) Involvement of the SMRT/NCoR-HDAC3 complex in transcriptional repression by the CNOT2 subunit of the human Ccr4-Not complex. Biochem J. 398, 461-467

13 Winkler, G. S., Mulder, K. W., Bardwell, V. J., Kalkhoven, E. and Timmers, H. T. (2006) Human Ccr4-Not complex is a ligand-dependent repressor of nuclear receptormediated transcription. Embo J. 25, 3089-3099

14 Meyer, S., Temme, C. and Wahle, E. (2004) Messenger RNA turnover in eukaryotes: pathways and enzymes. Crit Rev Biochem Mol Biol. 39, 197-216

15 Chen, J., Chiang, Y. C. and Denis, C. L. (2002) CCR4, a 3'-5' poly(A) RNA and ssDNA exonuclease, is the catalytic component of the cytoplasmic deadenylase. Embo J. 21, 1414-1426

16 Tucker, M., Staples, R. R., Valencia-Sanchez, M. A., Muhlrad, D. and Parker, R. (2002) Ccr4p is the catalytic subunit of a Ccr4p/Pop2p/Notp mRNA deadenylase complex in Saccharomyces cerevisiae. Embo J. 21, 1427-1436 
17 Mauxion, F., Faux, C. and Seraphin, B. (2008) The BTG2 protein is a general activator of mRNA deadenylation. Embo J. 27, 1039-1048

18 Bai, Y., Salvadore, C., Chiang, Y. C., Collart, M. A., Liu, H. Y. and Denis, C. L. (1999) The CCR4 and CAF1 proteins of the CCR4-NOT complex are physically and functionally separated from NOT2, NOT4, and NOT5. Mol Cell Biol. 19, 6642-6651

19 Chen, J., Rappsilber, J., Chiang, Y. C., Russell, P., Mann, M. and Denis, C. L. (2001) Purification and characterization of the 1.0 MDa CCR4-NOT complex identifies two novel components of the complex. J Mol Biol. 314, 683-694

20 Liu, H. Y., Badarinarayana, V., Audino, D. C., Rappsilber, J., Mann, M. and Denis, C. L. (1998) The NOT proteins are part of the CCR4 transcriptional complex and affect gene expression both positively and negatively. Embo J. 17, 1096-1106

21 Albert, T. K., Lemaire, M., van Berkum, N. L., Gentz, R., Collart, M. A. and Timmers, H. T. (2000) Isolation and characterization of human orthologs of yeast CCR4-NOT complex subunits. Nucleic Acids Res. 28, 809-817

22 Okazaki, N., Okazaki, K., Watanabe, Y., Kato-Hayashi, M., Yamamoto, M. and Okayama, H. (1998) Novel factor highly conserved among eukaryotes controls sexual development in fission yeast. Mol Cell Biol. 18, 887-895

23 Morita, M., Suzuki, T., Nakamura, T., Yokoyama, K., Miyasaka, T. and Yamamoto, T. (2007) Depletion of mammalian CCR4b deadenylase triggers elevation of the p27Kip1 mRNA level and impairs cell growth. Mol Cell Biol. 27, 4980-4990

24 Morel, A. P., Sentis, S., Bianchin, C., Le Romancer, M., Jonard, L., Rostan, M. C., Rimokh, R. and Corbo, L. (2003) BTG2 antiproliferative protein interacts with the human CCR4 complex existing in vivo in three cell-cycle-regulated forms. J Cell Sci. 116, 29292936

25 Dupressoir, A., Morel, A. P., Barbot, W., Loireau, M. P., Corbo, L. and Heidmann, T. (2001) Identification of four families of yCCR4- and Mg2+-dependent endonuclease-related proteins in higher eukaryotes, and characterization of orthologs of yCCR4 with a conserved leucine-rich repeat essential for hCAF1/hPOP2 binding. BMC Genomics. 2, 9

26 Walhout, A. J., Temple, G. F., Brasch, M. A., Hartley, J. L., Lorson, M. A., van den Heuvel, S. and Vidal, M. (2000) GATEWAY recombinational cloning: application to the cloning of large numbers of open reading frames or ORFeomes. Methods Enzymol. 328, 575592

27 Wessel, D. and Flugge, U. I. (1984) A method for the quantitative recovery of protein in dilute solution in the presence of detergents and lipids. Anal Biochem. 138, 141-143

28 Gobom, J., Nordhoff, E., Mirgorodskaya, E., Ekman, R. and Roepstorff, P. (1999) Sample purification and preparation technique based on nano-scale reversed-phase columns for the sensitive analysis of complex peptide mixtures by matrix-assisted laser desorption/ionization mass spectrometry. J Mass Spectrom. 34, 105-116

29 Mousson, F., Kolkman, A., Pijnappel, W. W., Timmers, H. T. and Heck, A. J. (2008) Quantitative proteomics reveals regulation of dynamic components within TATA-binding protein (TBP) transcription complexes. Mol Cell Proteomics. 7, 845-852

30 Paoletti, A. C., Parmely, T. J., Tomomori-Sato, C., Sato, S., Zhu, D., Conaway, R. C., Conaway, J. W., Florens, L. and Washburn, M. P. (2006) Quantitative proteomic analysis of distinct mammalian Mediator complexes using normalized spectral abundance factors. Proc Natl Acad Sci U S A. 103, 18928-18933

31 Backes, C., Keller, A., Kuentzer, J., Kneissl, B., Comtesse, N., Elnakady, Y. A., Muller, R., Meese, E. and Lenhof, H. P. (2007) GeneTrail--advanced gene set enrichment analysis. Nucleic Acids Res. 35, W186-192 
32 Rainer, J., Sanchez-Cabo, F., Stocker, G., Sturn, A. and Trajanoski, Z. (2006) CARMAweb: comprehensive R- and bioconductor-based web service for microarray data analysis. Nucleic Acids Res. 34, W498-503

33 Draper, M. P., Salvadore, C. and Denis, C. L. (1995) Identification of a mouse protein whose homolog in Saccharomyces cerevisiae is a component of the CCR4 transcriptional regulatory complex. Mol Cell Biol. 15, 3487-3495

34 Seimiya, H. and Smith, S. (2002) The telomeric poly(ADP-ribose) polymerase, tankyrase 1, contains multiple binding sites for telomeric repeat binding factor 1 (TRF1) and a novel acceptor, 182-kDa tankyrase-binding protein (TAB182). J Biol Chem. 277, 1411614126

35 Chang, P., Coughlin, M. and Mitchison, T. J. (2005) Tankyrase-1 polymerization of poly(ADP-ribose) is required for spindle structure and function. Nat Cell Biol. 7, 1133-1139

36 Aoki, T., Okada, N., Wakamatsu, T. and Tamura, T. A. (2002) TBP-interacting protein $120 \mathrm{~B}$, which is induced in relation to myogenesis, binds to NOT3. Biochem Biophys Res Commun. 296, 1097-1103

37 Persico, M., Ceol, A., Gavrila, C., Hoffmann, R., Florio, A, and Cesareni, G. (2005) HomoMINT: an inferred human network based on orthology mapping of protein interactions discovered in model organisms. BMC Bioinformatics. 6 Suppl 4, S21

38 Ito, T., Ota, K., Kubota, H., Yamaguchi, Y., Chiba, T., Sakuraba, K. and Yoshida, M. (2002) Roles for the two-hybrid system in exploration of the yeast protein interactome. Mol Cell Proteomics. 1, 561-566

39 Gavin, A. C., Bosche, M., Krause, R., Grandi, P., Marzioch, M., Bauer, A., Schultz, J., Rick, J. M., Michon, A. M., Cruciat, C. M., Remor, M., Hofert, C., Schelder, M., Brajenovic, M., Ruffner, H., Merino, A., Klein, K., Hudak, M., Dickson, D., Rudi, T., Gnau, V., Bauch, A., Bastuck, S., Huhse, B., Leutwein, C., Heurtier, M. A., Copley, R. R., Edelmann, A., Querfurth, E., Rybin, V., Drewes, G., Raida, M., Bouwmeester, T., Bork, P., Seraphin, B., Kuster, B., Neubauer, G. and Superti-Furga, G. (2002) Functional organization of the yeast proteome by systematic analysis of protein complexes. Nature. 415, 141-147

40 Yamashita, A., Chang, T. C., Yamashita, Y., Zhu, W., Zhong, Z., Chen, C. Y. and Shyu, A. B. (2005) Concerted action of poly(A) nucleases and decapping enzyme in mammalian mRNA turnover. Nat Struct Mol Biol. 12, 1054-1063

41 Bianchin, C., Mauxion, F., Sentis, S., Seraphin, B. and Corbo, L. (2005) Conservation of the deadenylase activity of proteins of the Cafl family in human. RNA. 11, 487-494 


\section{FIGURE LEGENDS}

\section{Figure 1 Integration of Flag/HA-tagged CNOT proteins in endogenous Ccr4-Not complexes}

(A) Cell extracts from stable human cell lines expressing the indicated tagged CNOT protein were analysed by immunoblotting using HA antibodies. Asterisks indicate epitope-tagged proteins. Detection of alpha-tubulin serves as a loading control. Control cells are HeLa S3 stably transfected with pBABE-puro. Molecular mass markers are indicated in kilo Daltons (kDa). (B) Some extracts from (A) were analysed by specific antibodies. Both endogenous and exogenous tagged proteins can be detected. (C) Immunoblot analyses of Flag/HA purifications of CNOT3L, CNOT9 or control cells using specific antibodies. (D) Silver stain of purified Ccr4-Not complexes. Asterisks indicate epitope-tagged CNOT3L, CNOT7 and CNOT9 protein bands, respectively from left to right. Molecular mass markers $(\mathrm{kDa})$ are given. (E) Cell extracts of CNOT3L, CNOT9 and control cell lines were Flag/HA-purified and analysed by immunoblotting using specific antibodies against TAB182. Flow through (FT) is also shown.

\section{Figure 2 Gene Ontology (GO) term overrepresentation clusters of Ccr4-Not} interactors

Interactors identified by at least 2 unique peptides and without peptides in a control purification (keratins excluded) were analysed for GO-term overrepresentation (see also Supplemental Figure 2). Colours represent the $\log 2 \mathrm{p}$-values of the Pearson correlation clustering multiplied by -1 on a scale of $0-35$. (A) Transcription cluster. (B) RNA stability cluster. (C) Splicing cluster. (D) RNA transport and localization cluster. (E) Nuclear envelope cluster. (F) Nuclear localization cluster.

\section{Figure $3 \quad$ Yeast two-hybrid interactions between CNOT proteins}

(A) A schematic representation of CNOT1 constructs is given. CNOT1N represents the Nterminal part (residues 1-716), CNOT1L contains the C-terminal part (residues 648-2376) and CNOT1C is a shorter C-terminal form (residues 1106-2376). (B) Yeast strain EGY48 was transformed with the indicated LexA- and B42-fusion expression plasmids together with a LacZ reporter gene containing eight LexA operator sites. Self-activation of LexA-CNOT2, LexA-CNOT4N, LexA-CNOT8 and LexA-CNOT9 fusions (data not shown) prevented their inclusion as DNA-binding domain fusions. Transformants were spotted on X-gal containing plates and incubated for two days (LexA-CNOT1L and LexA-CNOT1N) or for one day (LexA-CNOT3L, LexA-CNOT6, LexA-CNOT7 and LexA) at $30^{\circ} \mathrm{C}$. Blue colouring of yeast cells indicates binary interactions between LexA- and B42-fusion proteins.

\section{Figure 4 CNOT4 resides in a smaller protein complex}

Cell extracts were subjected to gel filtration under conditions of $150 \mathrm{mM} \mathrm{NaCl}$ and Ccr4-Not subunits were detected by specific antibodies. Input samples were loaded on the far left and right lanes, numbers indicate elution fractions. Calibration proteins are given in $\mathrm{kDa}$ with arrows: Blue Dextran (2000 kDa), Ferritin (450 kDa), Aldolase (158 kDa), BSA (67 kDa) and Ovalbumin $(45 \mathrm{kDa})$.

\section{Figure 5 CNOT6 interacts stronger with CNOT7 than with CNOT8}

(A) Cell extracts of $293 \mathrm{~T}$ cells with transient overexpressed deadenylases (left) or stable HeLa cell line extracts (right) were immunoblotted using antibodies directed against CNOT7 or CNOT8. Tagged (tag) or endogenous (endo) CNOT7 and CNOT8 proteins are indicated by arrows. (B) Extracts of cells containing epitope-tagged CNOT proteins or control cell extracts 
were affinity purified and analysed by specific antibodies. Asterisks indicate the crossreactivity of the CNOT8 antibody with the CNOT7 protein. (C) Cell extracts of CNOT6, CNOT2 or control cells were Flag-epitope affinity-purified under conditions of $150 \mathrm{mM} \mathrm{NaCl}$ (left of triangle) or $300 \mathrm{mM} \mathrm{NaCl}$ (right of triangle). Purified complexes were analysed by immunoblotting using specific antibodies. Asterisks indicate the cross-reactivity of the CNOT8 antibody with CNOT7 protein.

Figure 6 Model of human Ccr4-Not complexes differing in deadenylases

Stable core subunits are shown in grey ovals and the regulated CNOT4 subunit is given in white. Variable deadenylases between complexes are indicated in black, whereby the CNOT6/CNOT6L-CNOT8 interaction is less stable. 


\section{Table 1 Normalized spectral abundance factors of identified Ccr4-Not} components.

Flag/HA affinity-purified complexes of a tagged Ccr4-Not subunit were analysed by mass spectrometry. Control is HeLa S3 cell line stably transfected with pBABE-puro. Numbers represent 10-fold normalized spectral abundance factors (NSAFs, as described in [30]) for identified proteins. Molecular mass $(\mathrm{kDa})$ of these interactors are given on the right. Sum of unique peptides and sum of all identified peptides of identified proteins per purification are shown. Bold numbers indicate the NSAF values of deadenylase subunits in the purified Ccr4Not complexes using a tagged deadenylase.

\begin{tabular}{|c|c|c|c|c|c|c|c|c|c|c|}
\hline \multirow{2}{*}{$\begin{array}{l}\text { NSAF } \\
\text { Identified Protein }\end{array}$} & \multicolumn{9}{|c|}{ Tagged Protein } & \multirow[b]{2}{*}[\mathrm{kDa}]{} \\
\hline & CNOT 2 & CNOT $3 \mathrm{~L}$ & CNOT 6 & CNOT 7 & CNOT 8 & CNOT 9 & CNOT 10 & Control & CNOT $3 \mathrm{~S}$ & \\
\hline CNOT1 & 1.1 & 0.6 & 1.3 & 1.0 & 1.3 & 0.5 & 0.8 & 0 & 0.1 & 267 \\
\hline CNOT2 & 1.2 & 2.1 & 1.0 & 1.5 & 1.6 & 2.1 & 1.3 & 0 & 0 & 60 \\
\hline CNOT3 & 0.9 & 1.2 & 0.8 & 1.2 & 1.4 & 1.0 & 1.1 & 0 & 9.9 & 82 \\
\hline CNOT4 & 0 & 0 & 0 & 0 & 0 & 0.1 & 0.1 & 0 & 0 & 71 \\
\hline CNOT6 & 0 & 0.2 & 0.8 & 0.1 & $\mathbf{0}$ & 0.2 & 0 & 0 & 0 & 63 \\
\hline CNOT6L & 0.4 & 0.4 & $\mathbf{0}$ & 0.2 & $\mathbf{0}$ & 0.3 & 0 & 0 & 0 & 63 \\
\hline CNOT7 & 0.9 & 0.6 & 0.8 & 1.2 & $\mathbf{0}$ & 0.7 & 0 & 0 & 0 & 33 \\
\hline CNOT8 & 0.5 & 0.5 & $\mathbf{0}$ & $\mathbf{0}$ & 1.1 & 0.4 & 0.5 & 0 & 0 & 34 \\
\hline CNOT9 & 0.5 & 1.4 & 1.9 & 1.3 & 1.4 & 1.6 & 1.3 & 0 & 0 & 34 \\
\hline CNOT10 & 0.6 & 0.6 & 1.9 & 1.2 & 0.9 & 0.8 & 2.8 & 0 & 0 & 82 \\
\hline TAB182 & 3.1 & 1.9 & 0.8 & 1.6 & 1.8 & 1.9 & 0.8 & 0 & 0.1 & 182 \\
\hline C2ORF29 & 0.6 & 0.5 & 0.6 & 0.6 & 0.5 & 0.4 & 1.4 & 0 & 0 & 55 \\
\hline $\begin{array}{l}\text { Sum of } \\
\text { Unique Peptides }\end{array}$ & 131 & 347 & 97 & 279 & 13 & 258 & 56 & 0 & 15 & \\
\hline $\begin{array}{l}\text { Sum of All } \\
\text { Identified Peptides }\end{array}$ & 459 & 1970 & 138 & 1039 & & 638 & 101 & 0 & 52 & \\
\hline
\end{tabular}


Figure 1

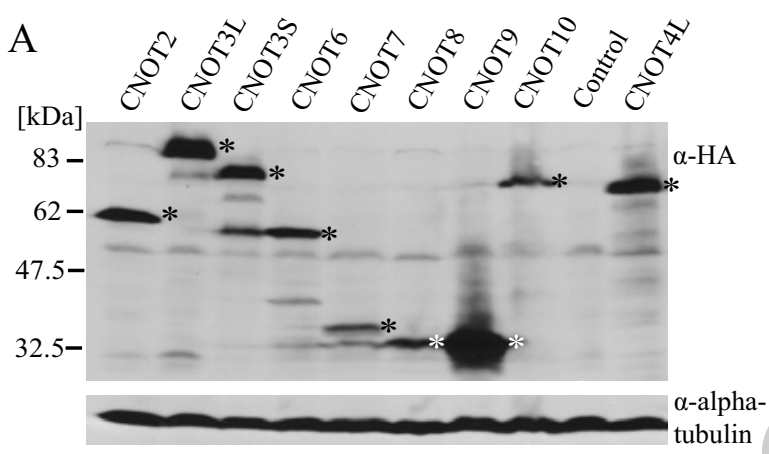

B

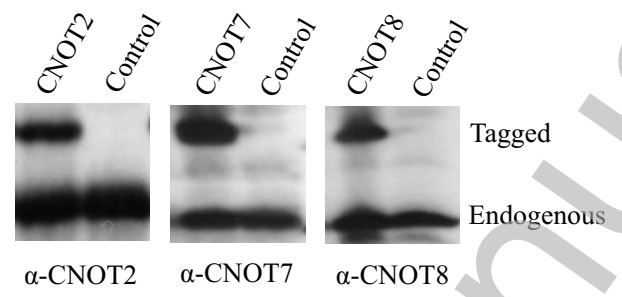

$\mathrm{C}$

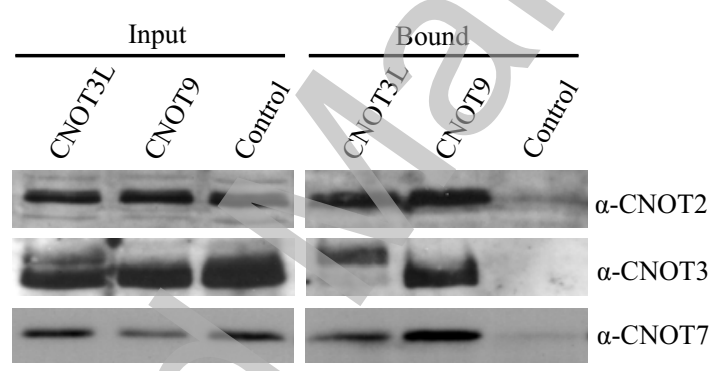

$\mathrm{D}$

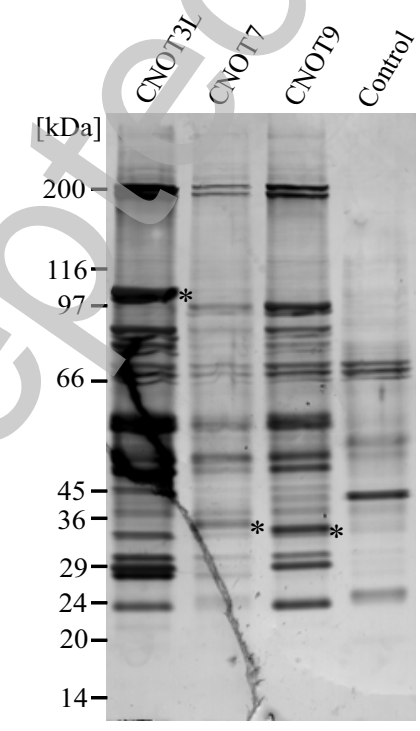

$\mathrm{E}$

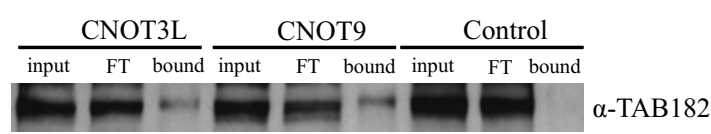


Figure 2

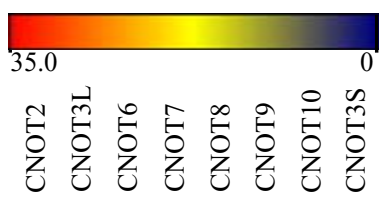

A

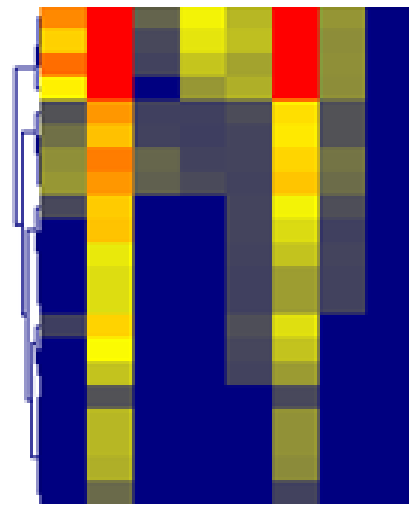

B

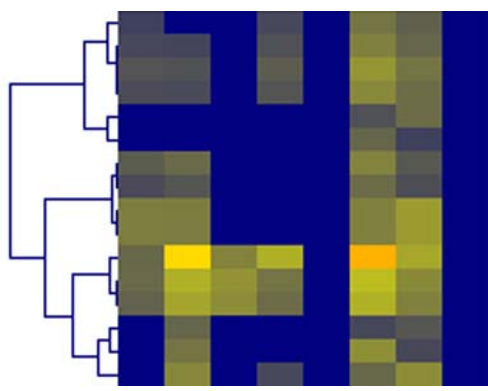

$\mathrm{C}$

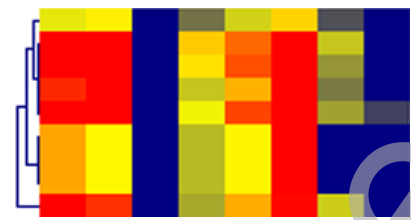

D

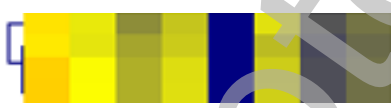

E

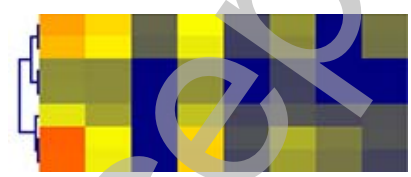

F

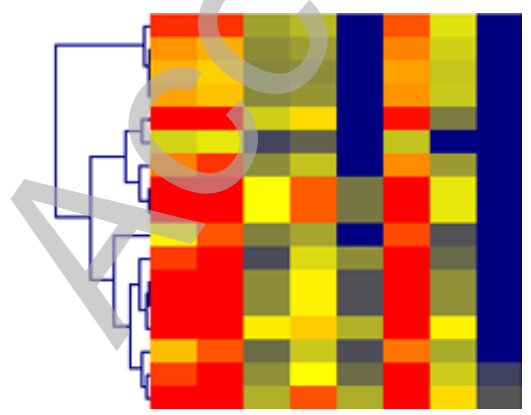

cellular biopolymer metabolic process gene expression

"nucleobase, nucleoside, nucleotide and nucleic acid metabolic process" RNA metabolic process

regulation of gene expression

regulation of macromolecule metabolic process

regulation of cellular metabolic process

regulation of metabolic process

"regulation of nucleobase, nucleoside, nucleotide and nucleic acid metabolic process" transcription

regulation of RNA metabolic process

RNA biosynthetic process

"transcription, DNA-dependent"

regulation of macromolecule biosynthetic process

regulation of transcription

"regulation of transcription, DNA-dependent"

DNA binding

DNA-directed RNA polymerase complex

nuclear DNA-directed RNA polymerase complex

RNA polymerase complex

RNA 3'-end processing

mRNA 3'-end processing

mRNA polyadenylation

RNA polyadenylation
low-density lipoprotein receptor binding

single-stranded DNA binding

cellular macromolecule catabolic process

macromolecule catabolic process

mRNA stabilization

RNA stabilization

mRNA binding

regulation of RNA stability

regulation of transcription from RNA polymerase II promoter

regulation of transcription fron

transcription from RNA polymerase II promoter

ribonucleoprotein complex

mRNA metabolic process

mRNA processing

RNA processing

RNA splicing

"nuclear mRNA splicing, via spliceosome"

"RNA splicing, via transesterification reactions"

"RNA splicing, via transesterification reactions with bulged adenosine as nucleophile" spliceosome

mRNA transport

"nucleobase, nucleoside, nucleotide and nucleic acid transport"

RNA localization

establishment of RNA localization

nuclear pore

nuclear import

protein import into nucleus

nuclear envelope

protein localization

establishment of protein localization

nuclear lumen

intracellular organelle lumen

membrane-enclosed lumen

organelle lumen

membrane part

nucleoplasm

intracellular organelle

organelle

nucleic acid binding

biopolymer metabolic proces

intracellular membrane-bounded organelle

membrane-bounded organelle

nucleus

etabolic proces

cellular metabolic process

cellular macromolecule metabolic process 
Figure 3

Lau

A

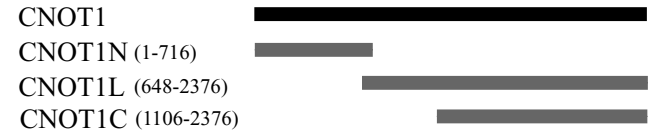

B

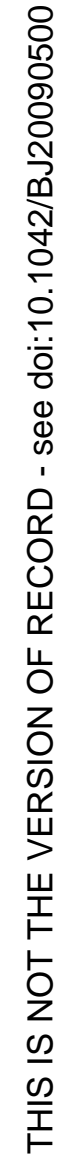

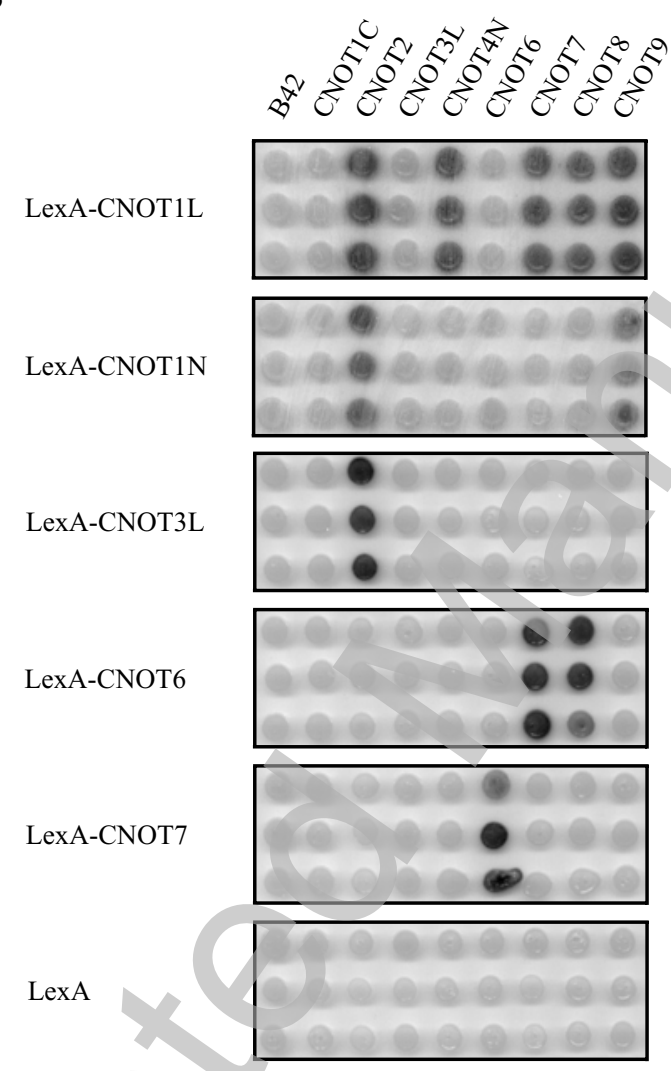

Licenced copy. Copying is not permitted, except with prior permission and as allowed by law. (C) 2009 The Authors Journal compilation (c) 2009 Portland Press Limited 
B Biochemical Journal Immediate Publication. Published on 26 Jun 2009 as manuscript BJ20090500

Figure 4

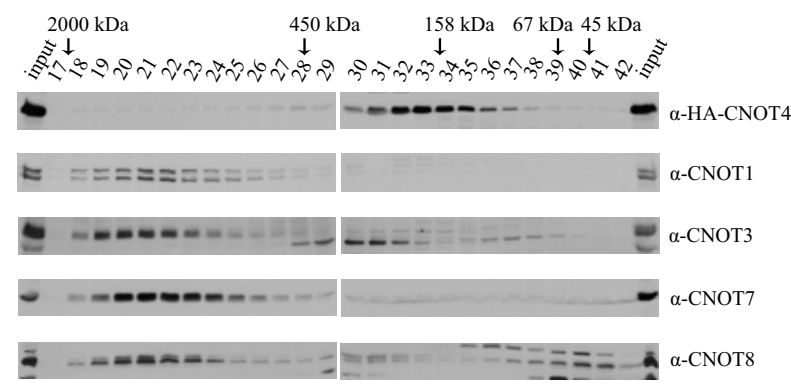


Figure 5

Lau

A Transient overexpression

Stable cell lines

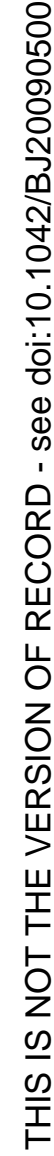

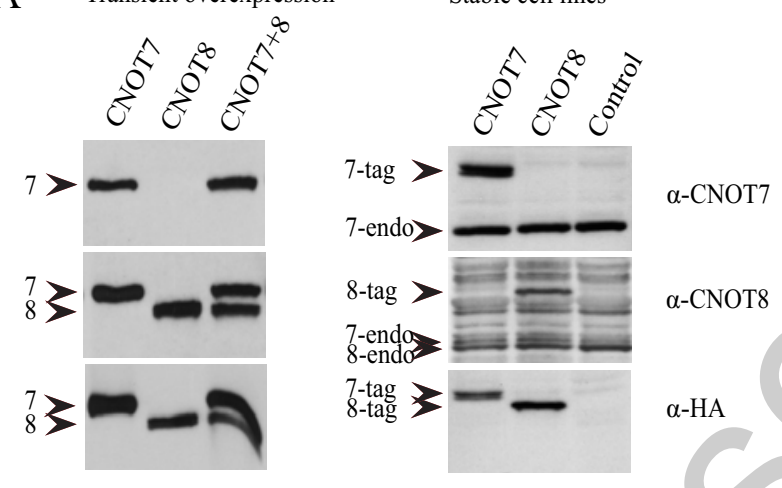

B
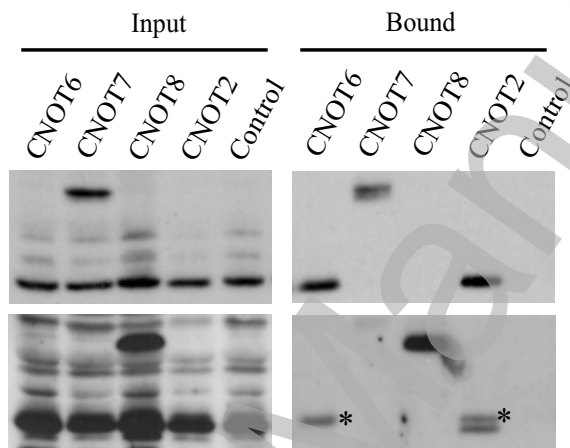

$\alpha-\mathrm{CNOT7}$

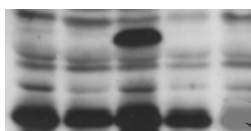

- * $\quad$ * *

$\alpha$-CNOT8

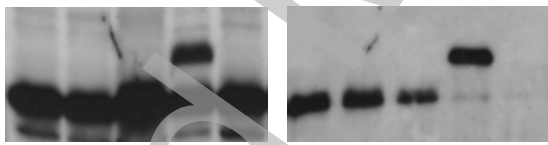

$\alpha-\mathrm{CNOT2}$

$\mathrm{C}$
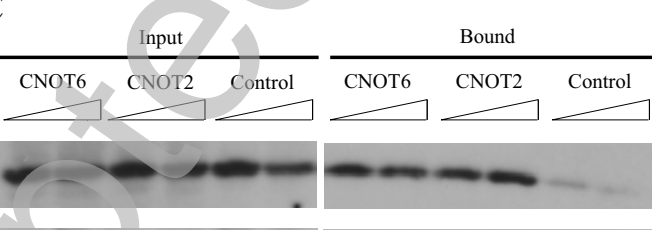

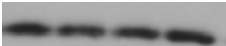

$\alpha-$ CNOT7

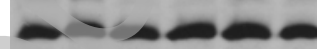

$\because * m * * 2 *$

$\alpha$-CNOT8

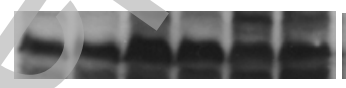

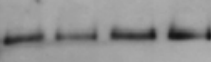

$\alpha$-CNOT3
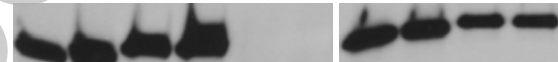

$\alpha-\mathrm{HA}$ 
Figure 6

CNOT7-CNOT6 complex

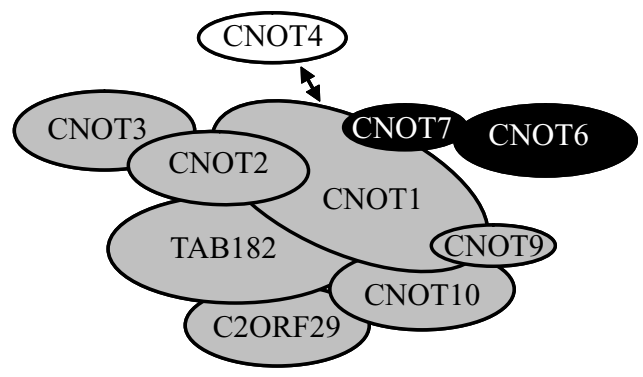

CNOT7-CNOT6L complex

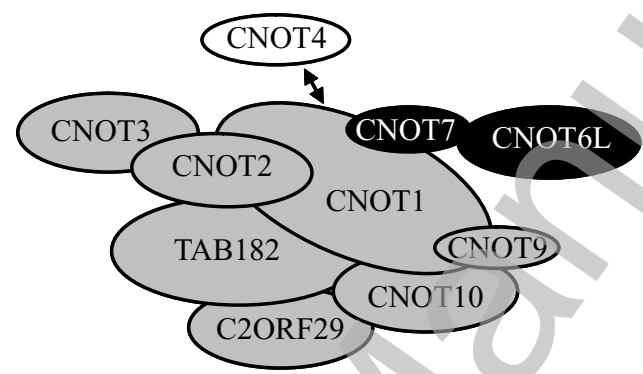

CNOT8-CNOT6 complex

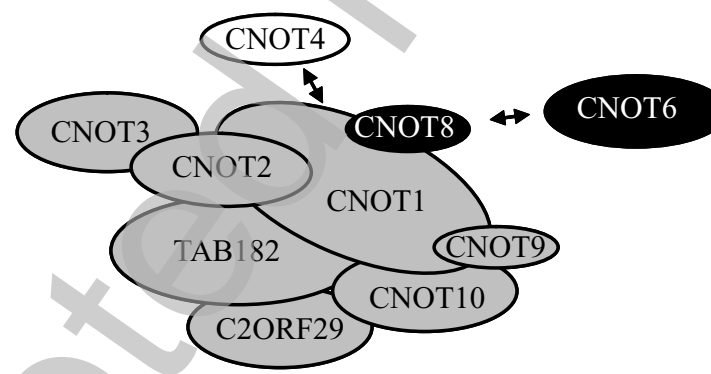

CNOT8-CNOT6L complex

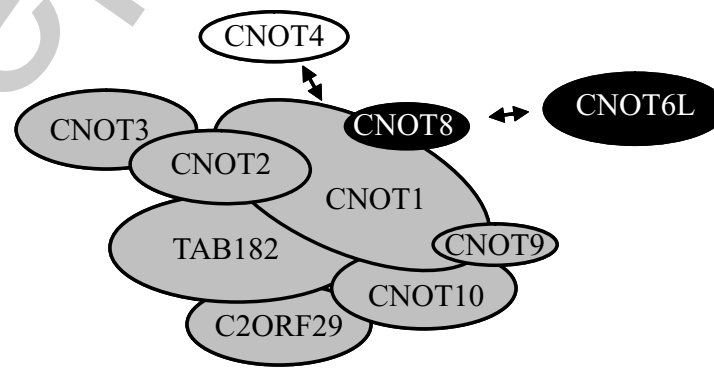

Licenced copy. Copying is not permitted, except with prior permission and as allowed by law. (C) 2009 The Authors Journal compilation (c) 2009 Portland Press Limited 